\title{
AVALIAÇÃO DA QUALIDADE DAÁGUA DA NASCENTE DO RIBEIRÃO DAS ANHUMAS (ARARAQUARA-SP) ATRAVÉS DO ESTUDO DOS MACROINVERTEBRADOS AQUÁTICOS
}

SAULINO, Hugo Henrique Lanzi. Centro Universitário de Araraquara - Uniara, Departamento de Ciências Biológicas e da Saúde, Araraquara-SP. E-mail: hugosaulino@ig.com.br.

CORBI, Juliano José. Programa de Mestrado em Desenvolvimento Regional e Meio Ambiente, Centro

Universitário de Araraquara - Uniara, Araraquara-SP.

CARACCIOLI, Leandro Carlos. Departamento de Hidrobiologia da Universidade Federal de São Carlos, São Carlos, SP.

\section{Resumo}

Nas últimas décadas, atividades antrópicas como a agricultura, lançamentos de efluentes industriaise domésticos e as áreas urbanas têm sido apontadas como as principais responsáveis pelas alterações e degradação dos ecossistemas aquáticos. Uma das formas de analisar a qualidade da água desses ambientes pode ser realizada por meio do estudo da fauna de macroinvertebrados aquáticos. O presente estudo teve como objetivo analisar a qualidade da água da nascente do ribeirão das Anhumas, através do estudo da comunidade de macroinvertebrados aquáticos. Foram realizadas duas coletas da fauna aquática, uma no mês de fevereiro e outra no mês de outubro de 2008. Foram escolhidos três trechos do córrego, onde se coletou duas amostras em cada trecho, totalizando 12 amostras. No total, foram coletadas 35 familias de macroinvertebrados, totalizando 644 indivíduos. A família Chironomidae foi dominante, com aproximadamente 57\% do total dos espécimes coletados. As métricas de avaliação da qualidade ambiental utilizando os macroinvertebrados apontaram para um bom estado de conservação da área de nascente do ribeirão das Anhumas, uma vez que foram encontradas uma alta riqueza de famílias e alta porcentagem de Ephemeroptera, Plecoptera e Trichoptera - EPT, em comparação com córregos de características semelhantes próximos a Araraquara. Os resultados apontampara a importância da conservação dessa área como referência de qualidade da água para a região de Araraquara.

Palavras-chave: Macroinvertebrados bentônicos; Córregos; Métricas de avaliação.

\begin{abstract}
In the last decades, anthropic activities such as agriculture activities, industrial and urban effluents and urban areas have been pointed as the main responsible for the alteration and degradation of the aquatic ecosystems. One way of analyzing the water quality of these environments is to study the aquatic microinvertebrated fauna. The present study aimed at analyzing the environmental water quality of Anhumas Stream in the city of Araraquara, SP, Brazil, by studying its aquatic macroinvertebrate community. Two samplings were carried out, being one in February and the other in October, 2008. Three sections of the stream were chosen for study, and two samples were collected at each site totalizing 12 samples. 35 families of macroinvertebrates were collected, totalizing 644 individuals. The Chironomidae family was the dominant one with approximately $57 \%$ of the total ofcollected organisms. The metrics of environmental evaluation using stream macroinvertebrates pointed to the conservation of the Anhumas stream, considering that we found a higher richness of families and a higher percentage of Ephemeroptera, Plecoptera and Tricoptera (EPT) than the ones found in streams with similar environments characteristics near to Araraquara region. The results pointed to the importance of conserving this area as a reference for water's quality in the region of Araraquara.
\end{abstract}

KEYwORDs: Benthic macroinvertebrates; Streams; Evaluation metrics. 


\section{INTRODUÇÃo}

O conhecimento ecológico dos ecossistemas lóticos integra os estudos das comunidades aquáticas e as características de variáveis físicas e químicas da água e do substrato. Esses habitats transitam desde ambientes de cabeceira e cursos de água de pequeno porte, como os riachos e córregos, até rios mais volumosos, localizados em trechos mais baixos da bacia de drenagem, e se caracterizam pelo movimento unidirecional em sentido à foz, diferindo grandemente em relação à profundidade, vazão, velocidade de corrente e tipo de substrato (SILVA, 2007). Os macroinvertebrados aquáticos são um grupo de extrema importância para a compreensão das estruturas desses ambientes (MORETTI; CALLISTO, 2005), pois têm uma grande influência no fluxo de energia do ecossistema e atuam nos processos de decomposição da matéria orgânica e ciclagem de nutrientes, como também são fontes de alimento para peixes e pássaros insetívoros (CARVALHO; UIEDA, 2004).

Por serem considerados bons bioindicadores da qualidade da água, sobretudo nos ambientes lóticos, em virtude de características específicas como sensibilidade, abundância, facilidade de coleta e identificação em nível de família, os macroinvertebrados aquáticos têm sido muito utilizados em diversos índices bióticos para a caracterização da qualidade biológica da água (LOT, 2007; SILVA, 2007). Esses índices são ferramentas de primeira grandeza para a avaliação da qualidade da água, uma vez que os parâmetros de referência constantes nas legislações são muitas vezes arbitrários, não levando em conta as diferentes capacidades de resistência dos diversos ambientes (ALBA-TERCEDOR, 1996).

Nesse contexto, o presente estudo tem como objetivo analisar a qualidade da água de uma área de preservação permanente na nascente do ribeirão das Anhumas, por meio do estudo da riqueza de macroinvertebrados aquáticos do local.

\section{Materiais E MÉTODOS \\ Área de estudo}

A nascente do ribeirão das Anhumas está localizada na fazenda Fortaleza (S 2186'962"WO 4801'111"), situada entre os municípios de Araraquara e Ibaté, no Estado de São Paulo. A propriedade é utilizada para manejo de florestas de eucalipto destinadas para a produção de celulose e papel, com uma área de preservação permanente correspondente a aproximadamente $2 \%$ da propriedade $(108,35$ hectares), com tipo de solo de característica arenosa na maior parte de extensão da fazenda.

A região apresenta precipitação média mensal de $122 \mathrm{~mm}$, sendo nos meses de novembro a março os períodos chuvosos e nos meses de maio a outubro os períodos de estiagem, com temperatura média anual de $26^{\circ} \mathrm{C}$ (Figuras 1 e 2 ). A vegetação da área de estudo é formada por floresta pantanosa e trechos em regeneração por reflorestamento de espécies nativas de Cerrado, visando atender à legislação que determina o tamanho da área de, no mínimo, 30 metros da área de preservação permanente, necessária para proteção dos rios com larguras de margens de até 10 metros. 


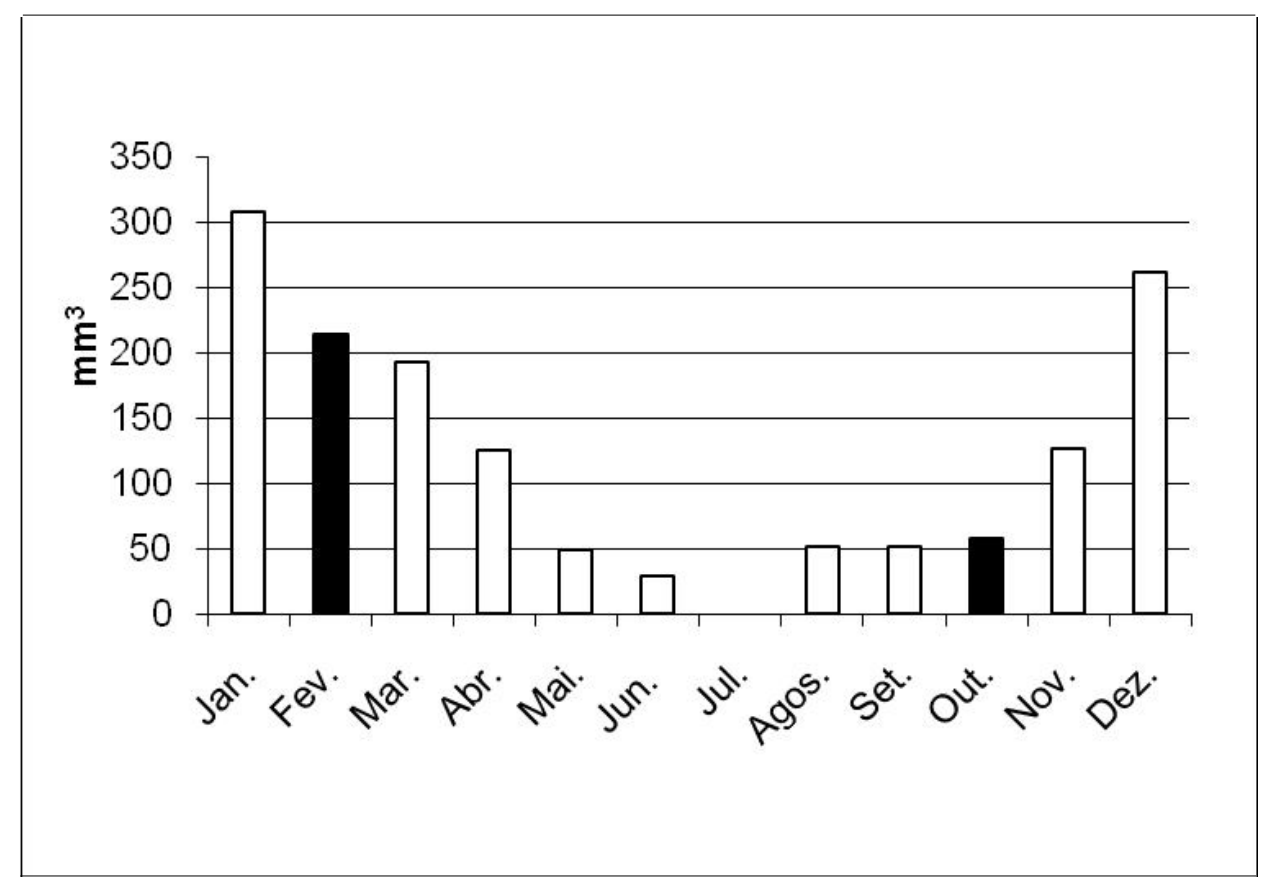

Figura 1 - Precipitação mensal no ribeirão das Anhumas no ano de 2008. Ribeirão das Anhumas, Araraquara-SP.

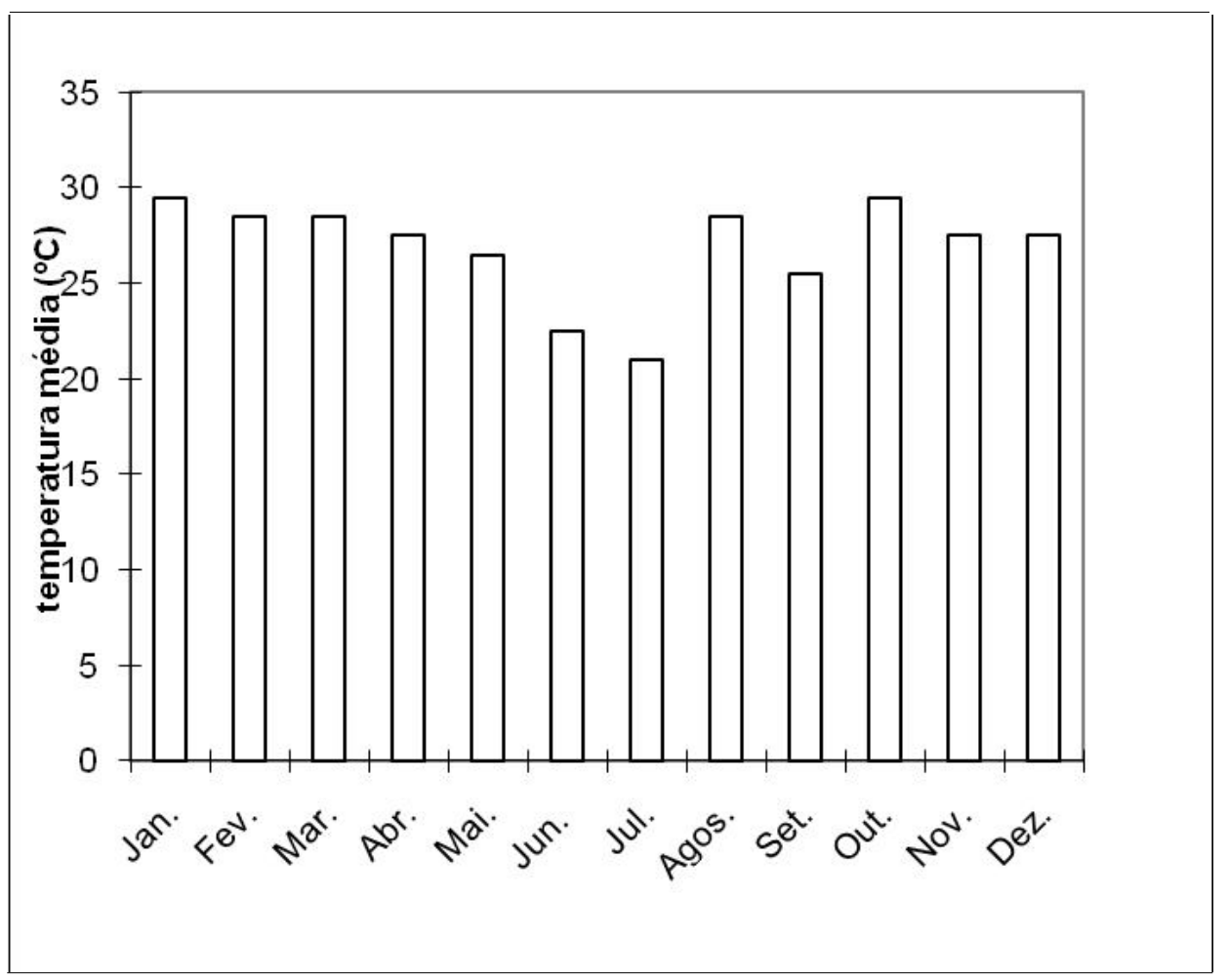

Figura 2 - Temperaturas médias dos meses de 2008 - ribeirão das Anhumas, Araraquara-SP. 
O local amostrado está inserido numa área onde ocorre a junção do córrego das Araras e o córrego Olho D'água, que nascem em uma propriedade vizinha e apresentam leito com menos de 10 metros de largura, formado por áreas de cabeceiras, profundidade menor que $15 \mathrm{~cm}$ e corredeiras com velocidades médias de $10 \mathrm{~m} / \mathrm{s}$. O substrato do canal é de característica arenosa, sem a presença de rochas. A vegetação do local amostrado é caracterizada pela presença de árvores de espécies nativas, arbustos e, em alguns trechos, de macrófitas aquáticas (Typha sp. e Eichhornia sp.).

\section{Variáveis físicas e químicas da água}

$\mathrm{Na}$ área selecionada do ribeirão das Anhumas foi caracterizada a vegetação local e tomadas medidas das principais variáveis físicas e químicas (largura, profundidade, temperatura, substratos predominantes, oxigênio dissolvido, $\mathrm{pH}$ e condutividade elétrica), seguindo o Protocolo de Caracterização Ambiental do projeto Biota-Fapesp para a padronização dos dados e caracterização ambiental do local de coleta (SURIANO, 2008). Para a caracterização das áreas de estudo foram utilizados equipamentos de campo como GPS, termômetro, oxímetro, pHmetro e condutivímetro, com o auxílio de aparelho multissensor Yellow Springs, modelo 556.

\section{Coleta e identificação dos macroinvertebrados aquáticos}

Com o objetivo de realizar uma avaliação da qualidade da água dessa área do ribeirão, foram realizadas duas coletas em meses diferentes do ano de 2008, uma em fevereiro (período de chuva) e outra em outubro (período de seca), a fim de se observar as mudanças na estrutura da comunidade de macroinvertebrados aquáticos. Em cada coleta, buscou-se explorar três trechos do córrego (englobando áreas de corredeira e remanso), de forma a representar com maior precisão a abundância e a riqueza da comunidade de macroinvertebrados aquáticos, respeitando a distância média de 20 metros entre os trechos de coleta. Em cada trecho foram escolhidos dois pontos próximos, totalizando seis amostras por coleta, obtendo um esforço amostral total de 12 amostras (Figuras 3 e 4 ).

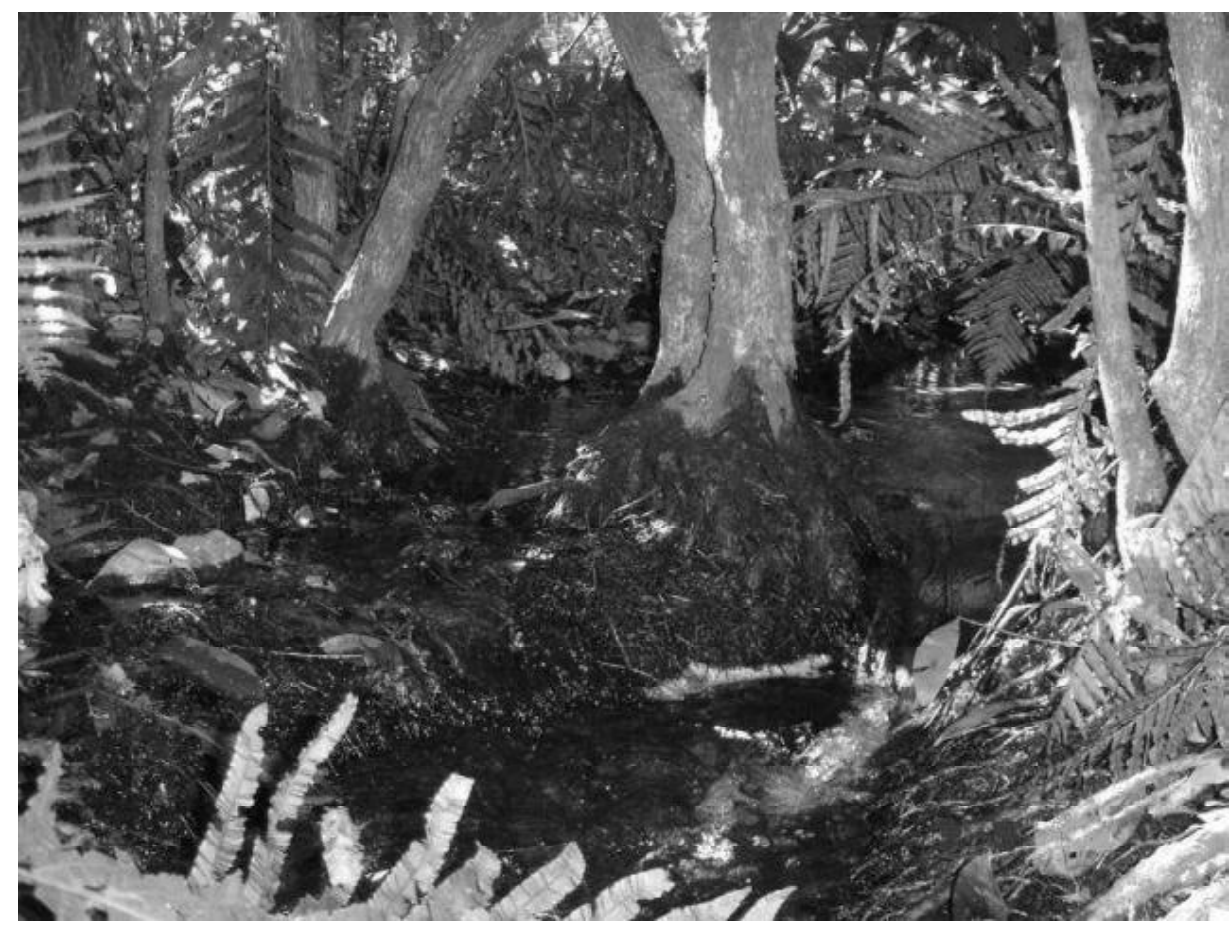

Figura 3 - Trecho de corredeira do ribeirão das Anhumas, Araraquara-SP. 


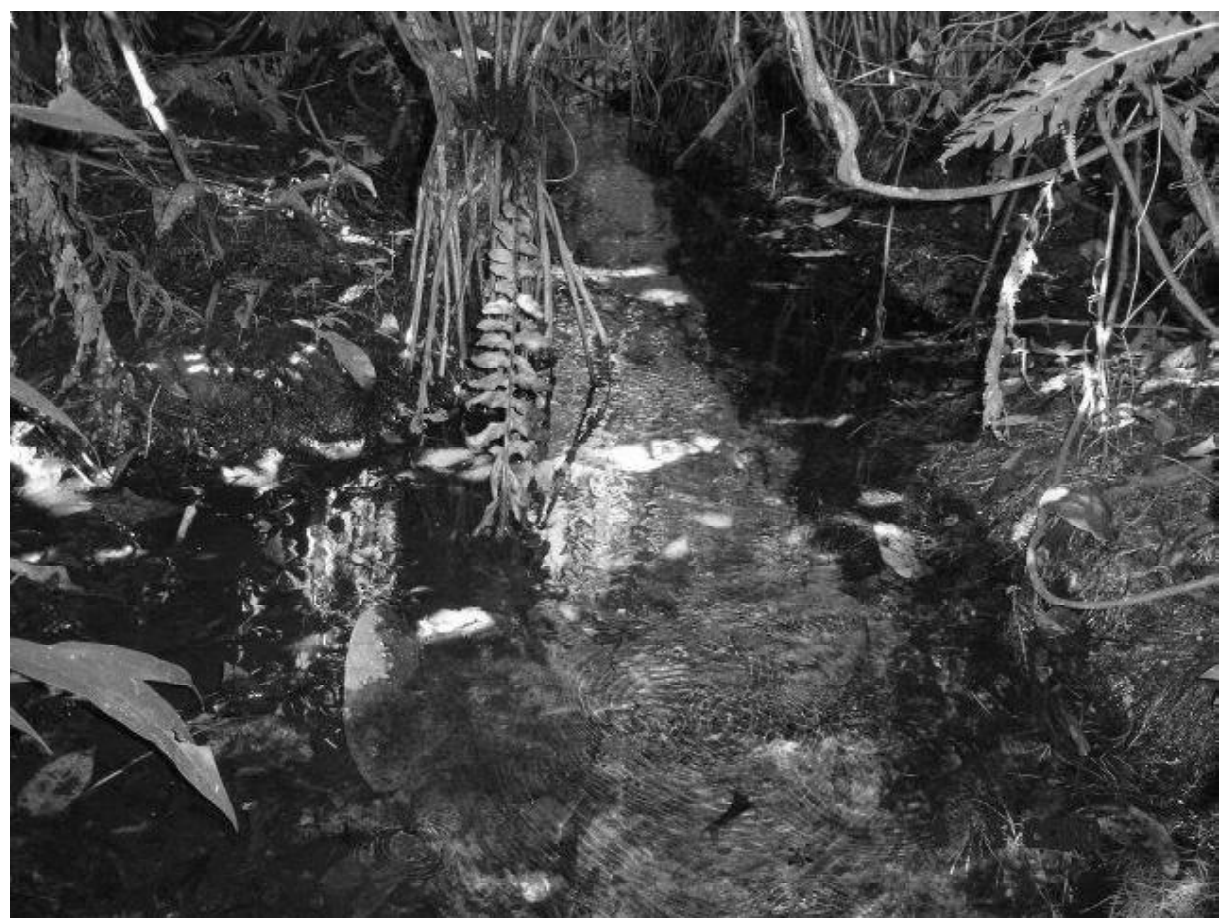

Figura 4 - Trecho de remanso do Ribeirão das Anhumas, Araraquara-SP.

A coleta da fauna de macroinvertebrados aquáticos de cada ponto foi realizada através de duas técnicas: método de varredura com o auxílio de uma rede em "D" (malha de 0,25mm de abertura), por um período de cinco minutos, procurando-se os vários biótipos do trecho, e pelo método de captura através de Surber (com malha de $0,25 \mathrm{~mm}$ ). O material coletado foi acondicionado em bujões plásticos de 15 litros e levado ao laboratório para o procedimento de triagem (FONTOURA, 1985).

A triagem dos macroinvertebrados foi feita em bandejas plásticas transluminadas e os espécimes foram conservados em frascos contendo álcool a 70\%. A identificação dos organismos, até o nível taxonômico de família, foi realizada utilizando lupa eletrônica com o uso de chaves de identificações especializadas (TRIVINHO-STRIXINO; STRIXINO, 1995; MERRITT; CUMMINS, 1996). Os resultados obtidos nos dois períodos sazonais foram analisados através dos valores de riqueza, abundância de indivíduos, diversidade de Shannon e dominância. Uma distribuição espacial entre os pontos das associações da estrutura da comunidade de macroinvertebrados amostrados foi avaliada com a aplicação da análise de escalonamento multidimensional (MDS), com matriz de similaridade calculada pelo índice de similaridade de Bray-Curtis. Para a avaliação biológica da qualidade da água foram utilizadas diferentes métricas de avaliação: porcentagem de organismos sensíveis EPT (Ephemeroptera, Plecoptera e Trichoptera), a razão entre o número de famílias de EPT e o número de famílias de macroinvertebrados (fam. EPT/Total de famílias X 100), Índice Biótico Biomonitoring Working Party System - BMWP (ALBA-TERCEDOR, 1988) e Índice Biótico Belga (IBB).

\section{Resultados E discussão}

\section{Variáveis físicas e químicas da água}

Os valores de $\mathrm{pH}$ variaram de próximo a 7 , no período chuvoso, a valores mais ácidos no período de estiagem (Tabela 1). A concentração de oxigênio dissolvido $(\mathrm{O} 2)$ apresentou-se elevada durante os períodos e nos pontos amostrados com médias de $8 \mathrm{mg} / \mathrm{L}$. A temperatura variou pouco: no período 
chuvoso apresentou média de $24,5^{\circ} \mathrm{C}$ e no período de estiagem, $21,5^{\circ} \mathrm{C}$. Os valores de condutividade foram baixos, porém variaram nos períodos amostrados, com o período de seca com valores mais altos se comparado com o período de chuvas (Tabela 1).

Tabela 1 - Variáveis físicas e químicas da água da nascente do ribeirão das Anhumas.

\begin{tabular}{|l|c|c|c|}
\hline \multicolumn{1}{|c|}{ Período } & Temperatura da água $\left({ }^{\circ} \mathbf{C}\right)$ & Condutividade $(\mathbf{m s} / \mathbf{c m})$ & pH \\
\hline Chuvoso & 24,5 & 0,05 & 6,91 \\
\hline Estiagem & 21,5 & 0,27 & 5,7 \\
\hline
\end{tabular}

Fonte: Dados de pesquisa.

Segundo Sperling (1998), em alguns ambientes o $\mathrm{pH}$ pode ser baixo por influências naturais, em decorrência da presença de ácidos húmicos e devido à decomposição da vegetação de origem alóctone. Vários fatores, como, por exemplo, a geologia da bacia de drenageme regimes de chuvas, podem influenciar na composição iônica de corpos d' água (ESTEVES, 1998). Acondutividade está relacionada com a função da concentração de íons presentes, portanto, quanto maior a concentração iônica, maior será a condutividade elétrica. Segundo Wantzen (2003), os córregos em áreas de cerrado são caracterizados por condutividade elétrica baixa e baixos valores de $\mathrm{pH}$, em razão da pobreza iônica dos solos. Dessa forma, a variação dos valores de $\mathrm{pH}$ e condutividade entre os períodos de chuva e de seca ocorreu, provavelmente, em decorrência das concentrações de íons originados da própria decomposição de matéria orgânica observada no canal do córrego. Em ecossistemas lóticos, as concentrações de oxigênio dissolvido são altas, em decorrência da baixa profundidade e de movimento constante, mesmo com ausência de plantas verdes (ODUM, 2001). Fatores relacionados com a velocidade da vazão, estação do ano, hora do dia e quantidade de materiais em suspensão podem interferir na temperatura da água (MAIER, 1978) - a variação de temperatura esteve relacionada com a própria temperatura ambiente, que é mais baixa no período de seca do que no chuvoso. Podemos inferir que as variações que ocorreram estão diretamente ligadas às características das próprias condições naturais do ambiente, uma vez que esses valores não são significativos de interferência antrópica no canal do córrego.

\section{Macroinvertebrados aquáticos}

No total, foram coletados 644 macroinvertebrados aquáticos no ribeirão das Anhumas, distribuídos em 35 famílias (Tabela 2). Os insetos compuseram a grande maioria dos animais amostrados, representando mais de $96 \%$ da fauna coletada, sendo que a família Chironomidae foi a mais representativa, com aproximadamente $57 \%$ do total de indivíduos (Figura 5). A família Leptophlebiidae também apresentou expressiva participação numérica com aproximadamente $9 \%$ do total. O conhecimento dos macroinvertebrados dos rios e riachos no Brasil ainda é escasso, e a identificação dos níveis de família ou gênero é mais comum atualmente em estudos de diversidade no País (MELO, 2006 apud CULLEN JR; VALLADARES-PADUA; RUDRAN, 2006). No entanto, vale ressaltar que a identificação, em nível de família, parece ser suficiente para avaliar os impactos de diferentes usos do solo em córregos localizados em áreas de Cerrado (CORBI; TRIVINHOSTRIXINO, 2006). 
Tabela 2 - Macroinvertebrados aquáticos coletados na nascente do ribeirão das Anhumas, Araraquara-SP.

\begin{tabular}{|c|c|c|c|c|}
\hline Ordem & família & Chuva & Seca & Total \\
\hline \multirow[t]{8}{*}{ Coleoptera } & Cerambycidae & 1 & & 1 \\
\hline & Curculionidae & & 2 & 2 \\
\hline & Dytiscidae & 5 & 8 & 13 \\
\hline & Elmidae & 1 & 7 & 8 \\
\hline & Helodidae & & 4 & 4 \\
\hline & Noteridae & & 2 & 2 \\
\hline & Ptilodactylidae & & 1 & 1 \\
\hline & Hydrophilidae & 1 & & 1 \\
\hline \multirow[t]{6}{*}{ Diptera } & Chironomidae & 138 & 232 & 370 \\
\hline & Ceratopogonidae & 6 & 1 & 7 \\
\hline & Culicidae & 1 & 1 & 2 \\
\hline & Ephidridae & & 1 & 1 \\
\hline & Sciomyzidae & 2 & & 2 \\
\hline & Simulidae & 3 & 5 & 8 \\
\hline \multirow[t]{4}{*}{ Ephemeroptera } & Baetidae & 9 & & 9 \\
\hline & Caenidae & 3 & & 3 \\
\hline & Leptophlebilidae & 56 & 1 & 57 \\
\hline & Leptohyphidae & 20 & 1 & 21 \\
\hline \multirow[t]{2}{*}{ Hemiptera } & Belostomatidae & 4 & 2 & 6 \\
\hline & Gerridae & & 2 & 2 \\
\hline Megaloptera & Corilidae & 1 & & 1 \\
\hline Leptoptera & Pyralidae & 1 & 1 & 2 \\
\hline \multirow[t]{5}{*}{ Odonata } & Libellulidae & 26 & 3 & 29 \\
\hline & Calopterygidae & & 3 & 3 \\
\hline & Aeshnidae & 7 & 2 & 9 \\
\hline & Coenagrionidae & 3 & & 3 \\
\hline & Gomphidae & 13 & 6 & 19 \\
\hline \multirow[t]{6}{*}{ Trichopetera } & Calamocera tidae & 5 & & 5 \\
\hline & Helycops ychidae & 5 & & 5 \\
\hline & Hydroptilidae & 2 & 3 & 5 \\
\hline & Leptoceridae & 5 & & 5 \\
\hline & Odontoceridae & 6 & & 6 \\
\hline & Philopotamidae & 2 & & 2 \\
\hline Plecoptera & Perlidae & & 7 & 7 \\
\hline Annelida & Naididae & 10 & 13 & 23 \\
\hline Total & & 336 & 308 & 644 \\
\hline
\end{tabular}

Fonte: Dados de pesquisa. 


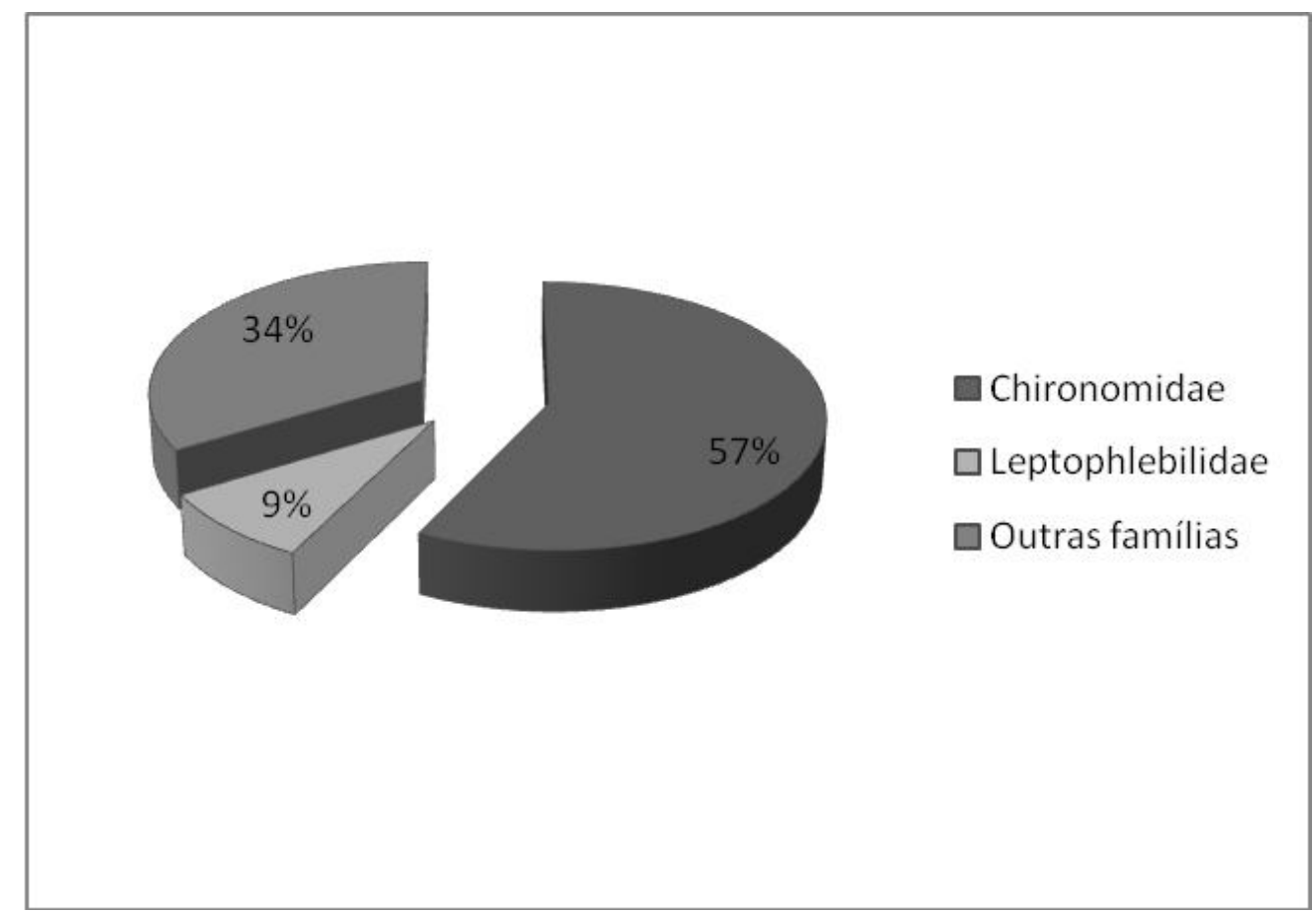

Figura 5 - Participação relativa das famílias de insetos aquáticos do Ribeirão das Anhumas, Araraquara, SP.

Os valores de riqueza e abundância não foram significantes entre os períodos amostrados ( $p>0,05)$. O período de chuva apresentou maior diversidade, caracterizado presença de maior número de famílias de algumas ordens, como Ephemeroptera, Trichoptera e Odonata. Esses organismos são frequentes em ambientes com maior fluxo de água e apresentam adaptações morfológicas que favorecem sua estabilização no ambiente, dificultando o arraste pela correnteza. O período de seca apresentou maior valor de dominância, sendo a família Chironomidae predominante nos dois períodos amostrados (Tabela 3). A grande porcentagem de insetos da família Chironomidae se deve aos aspectos de córregos do Estado de São Paulo, que se encontram no Bioma Cerrado e apresentam leitos arenosos e de baixa declividade, proporcionando baixa heterogeneidade ambiental (ROQUE; TRIVINO-STRIXINO, 2001). A análise de similaridade de Bray-Curtis demonstrou que os pontos do período chuvoso formaram agrupamentos bem definidos, demonstrando que estes foram mais similares do que os pontos amostrados no período de seca (Figura 6). Pode-se inferir que a maior pluviosidade acarreta aumento do fluxo de água no canal do córrego, promovendo mais habitat de corredeiras, o que também poderia explicar a presença de uma maior quantidade de famílias adaptadas às condições nesse período amostrado. De outra forma, com o baixo fluxo de água no período de seca, cresce a heterogeneidade de micro-habitats no córrego, com locais de remansos e corredeiras mais definidos, estabelecendo maiores distâncias de similaridade entre comunidades de macroinvertebrados. 
Tabela 3 - Índices aplicados à comunidade de macroinvertebrados aquáticos do Ribeirão das Anhumas, Araraquara nos períodos sazonais de chuva e seca de 2008.

\begin{tabular}{ccc}
\cline { 2 - 3 } & Chuva & Seca \\
\hline Táxons & 27 & 23 \\
Indivíduos & 336 & 308 \\
Dominância_D & 0,2116 & 0,5723 \\
Shannon_H' & 2,207 & 1,243 \\
\hline
\end{tabular}

Fonte: Dados de pesquisa.

Figura 6 - Análise de escalonamento multidimensional (MDS), com matriz de similaridade calculada pelo índice de similaridade de Bray-Curtis aplicada as comunidades de macroinvertebrados aquáticos do Ribeirão das Anhumas (Araraquara - SP) nos períodos de chuva e seca. P1C (ponto 1 chuva), P2C (ponto 2 chuva), P3C (ponto 3 chuva), P1S (ponto 1 seca), PS2 (ponto 2 seca), PS3 (ponto 3 seca). 
As métricas de avaliação ambiental utilizando os ribeirão das Anhumas, uma vez que os resultados macroinvertebrados detectaram um bom estado de conservação da área de preservação permanente do apontaram para uma alta riqueza de famílias e alta porcentagem de EPT (Tabelas 4 e 5).

Tabela 4 - Principais características comunitárias e métricas de avaliação da qualidade de água dos córregos Anhumas, Araraquara-SP.

\begin{tabular}{lc}
\hline \multicolumn{1}{c}{ Métricas } & Anhumas \\
\hline Número de famílias -F & 35 \\
EPT (\%) & 19 \\
(no fam. EPT/total fam.) X 100 & 31 \\
Shannon_H (diversid ade) & 2,2 \\
Margalef (riqueza) & 7,6 \\
Equid ade & 0,6 \\
Índice Biótico (BMWP) & 104 \\
Índice Biótico Belga (IB B) & 10 \\
\hline
\end{tabular}

Fonte: Dados de pesquisa.

Tabela 5 - Faixas de valores dos índices bióticos (IBB e BMWP) utilizados para o cálculo da qualidade da água.

\begin{tabular}{cccc}
\hline Classe & Faixa de "score" IBB & Faixa de "score" BMWP & Qualidade da água \\
\hline 1 & $9-10$ & $=81$ & Excelente \\
2 & $7-8$ & $80-61$ & Boa \\
3 & 6 & $60-41$ & Regular \\
4 & $4-5$ & $40-26$ & Ruim \\
5 & $=3$ & $=26$ & Péssima
\end{tabular}

Fonte: Corbi (2006). 
Os resultados obtidos das métricas de riqueza, BMWP e IBB foram semelhantes quando comparados com outros estudos realizados em córregos de mesma classificação, e com características ambientais semelhantes, na região de São Carlos e Araraquara (CORBI, 2006; PAULA; FONSSECA-GESSNER, 2010). Diversos estudos apontam que as matas ciliares são consideradas os mais importantes componentes na estruturação das comunidades de macroinvertebrados em sistemas lóticos de baixa ordem (VANNOTE et al, 1980; GREGORY et al., 1991; CORBI, 2006), uma vez que fornecem material alóctone vegetal (troncos, folhas, frutos) (BUNN, et al., 1999), servindo de recursos para numerosos invertebrados detritívoros.

Alguns estudos (ROQUE et al., 2000; ALLAN, 2004; CORBI, 2006; SURIANO, 2008) demonstram que locais onde há ausência desse tipo de vegetação são habitados por uma fauna de macroinvertebrados mais homogênea e pobre; isso decorre do aumento da biomassa de perifíton, da maior incidência de luz e da redução da quantidade dos restos de vegetais em decomposição, ao contrário de córregos de áreas florestadas, que apresentam uma fauna mais rica e heterogênea.

\section{Conclusões}

Após a análise da estrutura da comunidade de macroinvertebrados aquáticos, pode-se concluir que a água da nascente do ribeirão das Anhumas se encontra em um bom estado de conservação, o que demonstra a importância da conservação das áreas de preservação permanente para a manutenção da riqueza de macroinvertebrados aquáticos e para a qualidade ambiental desses ambientes. No entanto, atividades de educação ambiental associadas a práticas adequadas de preservação das matas ciliares e das áreas de ecótone podem contribuir para a manutenção e o aumento da riqueza de espécies do local, em consequência da melhor conservação desse ecossistema.

\section{REFERÊNCIAS}

ALBA-TERCEDOR, J. Macroinvertebrados acuaticos y calidad de las aguas. Simposio delAgua en Anadalucia, 4., 1996. Actas. v.2, p.203-213.

CARVALHO, E. M.; UIEDA, V. S. Colonização por macroinvertebrados bentônicos em substrato artificial e natural em um riacho da serra de Itatinga, São Paulo. Revista Brasileira de Zoologia, v. 21, n. 2, p. 287-293, 2004.

CLETO-FILHO, S.E.N.; WALKER, I. Efeitos da ocupação urbana sobre a macrofauna de invertebrados aquáticos de um Igarapé da Cidade de Manaus/AM - Amazônia Central. Acta Amazônica. v.31, n.1, p. 69-89, 2001.

BUNN, S. E.; DAVIES, P.; MOISCH, T. D. Ecosystem measures of river health and their response to reparian and catchment degradation. Freshwater biology, v. 41, p.333-345, 1999.

\section{CORBI, J.J. Influência espacial de manejo de} solo sobre os macroinvertebrados aquáticos de córregos: ênfase para o cultivo de cana-deaçúcar em áreas adjacentes. 2006. 96 f.. Tese (Doutorado) - Departamento de Hidrologia/ Laboratório de Entomologia Aquática) Universidade Federal de São Carlos, São Carlos, 2006.

CORBI, J.J.; TRIVINHO-STRIXINO, S. Influence of taxonomic resolution of stream macroinvertebrate communities on the evaluation of different land uses. Acta Limnologica Brasiliensia, v.18, n.4, p.469475, 2006.

CORBI, J.J.; TRIVINHO-STRIXINO, S.; DOS SANTOS, A. Environmental evaluation of metals in sediments and dragonflies due to sugar cane cultivation in Neotropical streams. Water, Air and Soil Pollution, v.195, p. 325-333, 2008.

CORBI, J.J. et al. A. Bioaccumulation of metals in aquatic insects of streams located in areas with sugar 
cane cultivation. Quimica Nova, v.33, n.3, p. 644648, 2010.

ESTEVES, F.A. Fundamentos de Limnologia. 2.ed.. São Paulo: Interciência. 1988. 601p.

FONTOURA, A.P. Manual de vigilância da qualidade das águas superficiais. Avaliação biológica da qualidade da água. Porto: Instituto de zoologia. Faculdade de Ciências - Universidade do Porto, 1985. 38p.

GREGORY, S. V.; SWANSON, F. J.; MCKEE, W. A.; CUMMINS, K. W. An ecosystem perspective of riparian zones. Bioscience, v.41, p. 333-345.

LOT, A. Caracterização da qualidade de água do Ribeirão das Cruzes-Araraquara (SP) através de variáveis físicas e químicas e dos macroinvertebrados bentônicos. 2007. Tese (Mestrado). Centro Universitário de Araraquara, Araraquara, 2007.

MAIER, M.H. Considerações sobre caracteristicas limnologicas de ambientes lóticos. Boletim do Instituto de pesca, São Paulo, v. 5, n. 2, p.75-90, 1978.

MEDEIROS, M.B. Caracterização das comunidades de macroinvertebrados bentônicos da bacia do córrego Riacho Fundo, Brasília-DF, e uso destas como bioindicadores de qualidade de água.1997. 82f. Dissertação (Mestrado) Universidade de Brasília-DF, 1997.

MELO, A.S. Diversidade de macroinvertebrados, In: CULLEN JR, L.; VALLADARES-PADUA, C; RUDRAN, R. Métodos de estudos em biologia da conservação e manejo da vida silvestre. Cuiritiba: UFPR, 2006. Cap. 2-3

MERRITT, R.W.; CUMMINS, K.W. An Introduction to the Aquatic Insects of North
America. Dubuque, Iowa: Kendall-hunt, 1996.

MORETTI, M.S.; CALLISTO, M. Biomonitoring of benthic macroinvertebrates in the middle Doce River watershed. Acta Limnologica Brasiliensia, v. 17, n. 13, p. 267-281, 2005.

ODUM, E. Ecologia. Rio de Janeiro: Guanabara Koogan, 2. ed., 1988. 433 p.

ODUM, E.P. Fundamentos em ecologia. Fundação Calouste Gulbenkian, 2001. 927p.

PAULA, M.C.; FONSECA-GESSNER, A.A. Macroinvertebrates in low-order streams in tow fragments of Atlantic Forest in different states of conservation, in the State of São Paulo (Brazil). Brazilian Journal of Biology, v.70, n. 3, p. 899909, 2010.

ROQUE, F.O.; TRIVINO-STRIXINO, S. Bentic macroinvertebrates in mesohabitats of diferents spatial dimensions in a first order stream (São Carlos -SP). Acta Limnologica Brasiliensia, v.13, n.2, p. 69-77, 2001.

SILVA, N.T.C. Macroinvertebrados bentônicos em áreas com diferentes graus de preservação ambiental na Bacia do Ribeirão Mestre d' armas, DF. 2007. 113f. Dissertação de (Mestrado) Departamento de Ecologia, Universidade de Brasília) - Brasília, 2007.

SPERLING, E.V. Qualidade da água em atividade de mineração. In: DIAS, L. E.; MELO, J. W. V. Ed. Recuperação de áreas degradadas. Viçosa: Viçosa, 1998 p. 95-115.

SURIANO, M.T. Macroinvertebrados em córregos de baixa ordem sob diferentes usos do solo no Estado de São Paulo: subsídios para o biomonitoramento. 2008. 145 f. Tese (Doutorado) - São Carlos: Departamento de Hidrologia/ 
Laboratório de Entomologia Aquática) -

Universidade Federal de São Carlos, 2008.

VANNOTE, R. L. MINSHALL, G. W.;

CUMMINS, K. W.; SEDELL, J. R. CUSHING, C.

E. The river continuum concept. Can. J. Fish.

Aquat. Sc. v.37, p. 130-137, 1980.

WANTZEN, K.M. Cerrado stream characteristic of a treathened freshwater ecosystem type on the
Teatiary Shields of Central South America.

Amazoniana, v. 3, n. 4, p.481-502. 2003.

WETZEL, R.G. Limnology. Philadelphia. Sawders ed. 1983. 743p.

TRIVINHO-STRIXINO, S.; STRIXINO, G. Larvas de Chironomidae (Diptera) do Estado de São Paulo: Guia de Identificação e Diagnose dos Gêneros. São Carlos: UFSCar, 1995. 227p.

RECEBIDO EM 22/11/2010

ACEITO EM 28/6/2011 